\title{
Seven newly recorded species and families of spiders from Reunion Island (Malagasy region) (Araneae, Araneomorphae)
}

\author{
by Maxime JACQuot ${ }^{1,2}$, Brice Derepas ${ }^{1} \&$ Jean-Philippe Deguine ${ }^{1}$ \\ ${ }^{1}$ CIRAD, UMR PVBMT, F - 97410 Saint-Pierre, Réunion, France \\ < jacquot.maxime.a@gmail.com><jean-philippe.deguine@cirad.fr> <bricederepas24@gmail.com> \\ ${ }^{2}$ Université de La Réunion, UMR PVBMT, F - 97410 Saint-Pierre, Réunion, France
}

\begin{abstract}
To date, 24 spider families have been identified on Reunion Island. But published data on spider fauna mainly concerned natural habitats and highlands. Studies of predatory arthropods in agroecosystems provided the opportunity to conduct a thorough investigation of spider diversity. Seven species and families of spiders are newly recorded for Reunion Island: Creugas gulosus Thorell, 1878 (Corinnidae), Oedignatha scrobiculata Thorell, 1881 (Liocranidae), Ero aphana (Walckenaer, 1802) (Mimetidae), Palicanus caudatus Thorell, 1897 (Miturgidae), Oecobius marathaus Tikader, 1962 (Oecobiidae) and Orthobula impressa Simon, 1897 (Phrurolithidae) and Tropizodium peregrinum Jocqué \& Churchill, 2005 (Zodariidae).

Résumé. - Sept espèces et familles d'Araignées nouvelles pour la Réunion (Araneae, Araneomorphae). Jusqu'à aujourd'hui, 24 familles d'Araignées avaient été recensées à l'île de la Réunion. Ces données publiées sur l'arachnofaune ont principalement concerné les habitats naturels et d'altitude. Des inventaires des arthropodes prédateurs dans les agroécosystèmes ont permis de compléter les connaissances sur la diversité des Araignées. Ainsi, sept espèces et familles d'Araignées nouvelles pour la Réunion ont été recensées : Creugas gulosus Thorell, 1878 (Corinnidae), Oedignatha scrobiculata Thorell, 1881 (Liocranidae), Ero aphana (Walckenaer, 1802) (Mimetidae), Palicanus caudatus Thorell, 1897 (Miturgidae), Oecobius marathaus Tikader, 1962 (Oecobiidae) and Orthobula impressa Simon, 1897 (Phrurolithidae) et Tropizodium peregrinum Jocqué \& Churchill, 2005 (Zodariidae).
\end{abstract}

Keywords. - Faunistics, Mascarene Islands, new records, agroecosystems.

Reunion is a volcanic island located in the south western Indian Ocean. As most major islands in the region, Reunion was colonized mainly by animals and plants from Madagascar; some lineages with high dispersal abilities came from biogeographical regions of the southern hemisphere (AgNARSSON \& KunTNER, 2012). The first, and important, study of spider fauna on Reunion Island was conducted by VINSON (1863), who identified species belonging to 17 families. It was not until the end of the $\mathrm{xx}^{\text {th }}$ century that four families were newly recorded: Trochanteridae (Schmidt \& Jocqué, 1983), Sicariidae and Hahniidae (Lopez, 1990) and Nesticidae (Ledoux \& HALlÉ, 1995). During the 2000s, three families were recorded: Linyphiidae [first record by Quilici et al. (2003)], Ctenidae (Ledoux, 2004) and Oonopidae [first record by Platnick et al. (2011)].

Up to this day, there are 24 spider families known on Reunion Island. Previous studies focussed on natural habitats and most were conducted in the Reunion highlands. A study of predatory arthropods in horticultural agroecosystems aimed at developing an insecticides free cropping system gave us the opportunity to undertake a thorough investigation of spider diversity.

\section{Material AND METHODS}

Samples were collected on the west coast of Reunion Island mainly in mango orchards, as part of the BIOPHYTO project (www.biophyto.org). Photographs were taken by Brice Derepas with a Nikon AZ100 Multizoom and a DS-Fi2 5-megapixel camera using focus stacking techniques with NIS-Elements software. Photographs of the vulva were taken after dissection and a few minutes in $5.2 \%$ sodium hypochlorite solution $(\mathrm{NaClO})$ to ensure digestion of unsclerotized 
tissue. Voucher specimens have been deposited in two permanent arthropod collections. Voucher codes beginning by BDER correspond to samples identified by Brice Derepas and Maxime Jacquot and deposited in the collection of UMR PVBMT (Saint-Pierre, Reunion, France). Voucher codes beginning by MRAC correspond to samples identified by Arnaud Henrard and deposited in the collection of the Musée Royal de l'Afrique Centrale (Tervuren, Belgium). Voucher codes marked with an asterisk $(*)$ indicated photographed specimens.

\section{RESULTS}

We found seven new spider families and species that have previously not been known to occur on Reunion Island. We detail collection data and provide photographs of these species.

Family Corinnidae Karsch, 1880

Genus Creugas Thorell, 1878

Creugas gulosus Thorell, 1878 (fig. 1)

Creugas gulosus Thorell, 1878: 175; SAARISTO, 2002: 6, fig. 11-15; SAARISTO, 2010: 58, fig. 6.1-5; RAVEN, 2015 : fig. 9a-d (female), 10a-d (male).

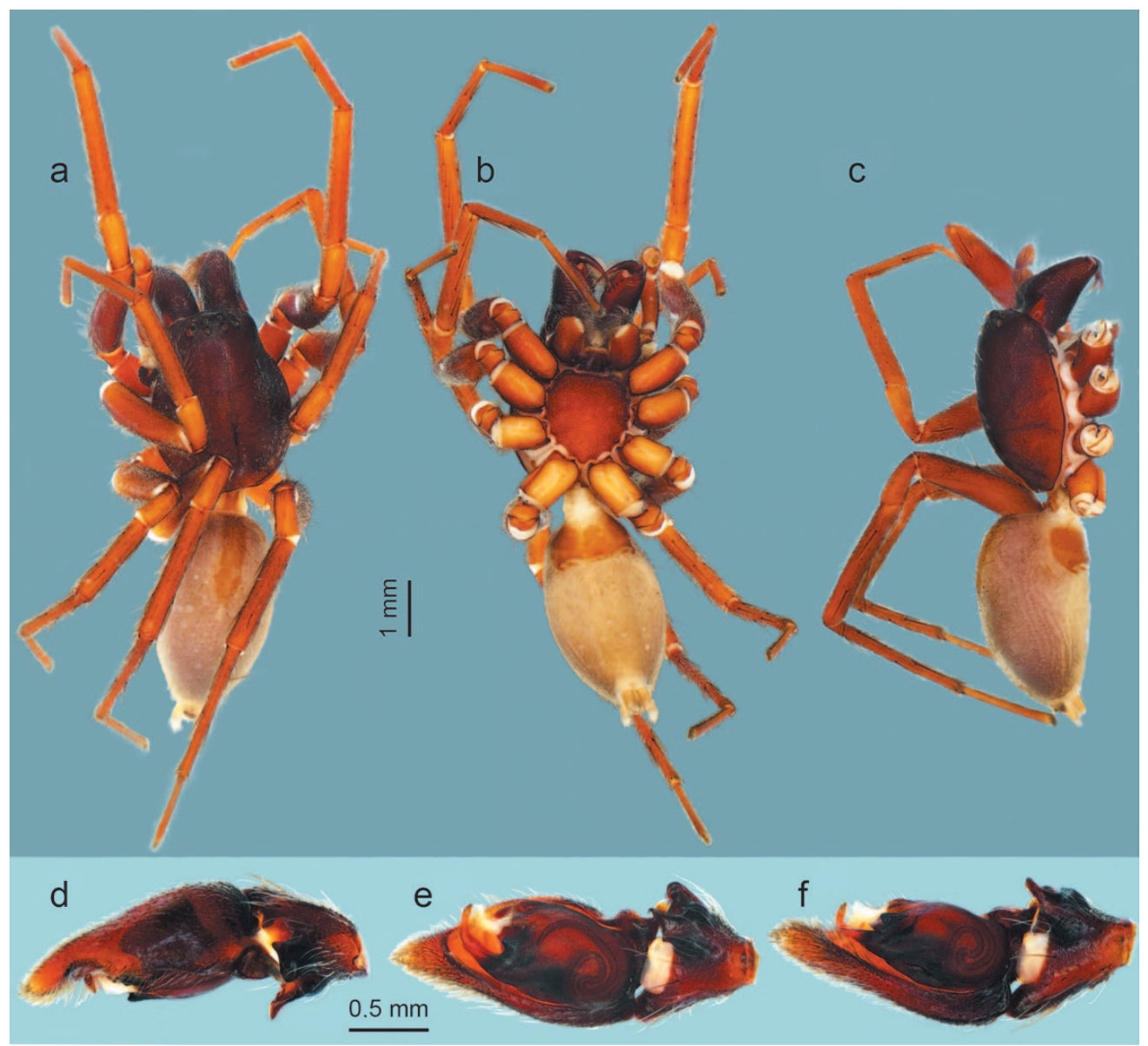

Fig. 1. - Creugas gulosus Thorell, ô. - a, Dorsal view. - b, Ventral view. - c, Lateral view. - d, Palp, retrolateral view. - e, Palp, ventral view. - f, Palp, prolateral view. 
Material examined.-Reunion Island. 1 §̂,Étang-Salé(alt. 110 m,29.VIII.2012(BDER00372_0101*), M. Jacquot et al. leg. (pitfall traps).

Distribution. - Pantropical, recorded in Seychelles and newly recorded from Reunion Island.

Family Liocranidae Simon, 1897

Genus Oedignatha Thorell, 1881

Oedignatha scrobiculata Thorell, 1881 (fig. 2)

Oedignatha scrobiculata Thorell, 1881: 209; Deeleman-Reinhold, 2001: 267, fig. 348-356; SAaristo, 2002: 9, fig. 18, 20; SAARISTO, 2010: 61, fig. 6.8, 6.10 .

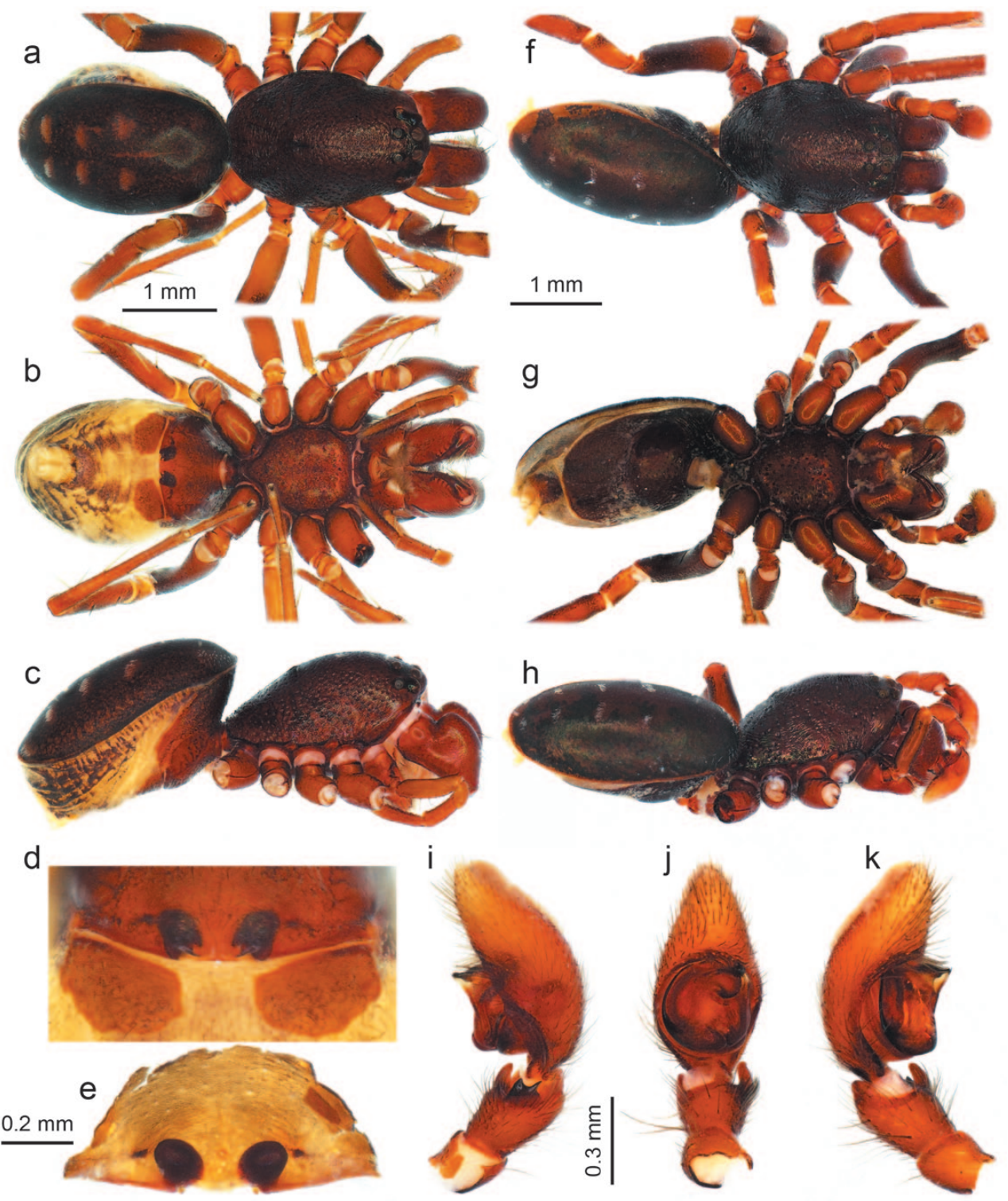

Fig. 2. - Oedignatha scrobiculata Thorell. - a-e,, : a, dorsal view; b, ventral view; c, lateral view; d, epigyne; e, vulva. $\mathrm{f}-\mathrm{k}, \widehat{\jmath}$ : f, dorsal view; g, ventral view; h, lateral view; i, palp, retrolateral view; j, palp, ventral view; $\mathrm{k}$, palp, prolateral view. 
Material examined. - Reunion Island. 6 +, Saint-Leu, chemin des Bois-de-Lait (alt. $270 \mathrm{~m}$ ), 25.VIII.2009, 4.III.2010, 18.VII.2011, 1.VI.2012 (BDER00379_0101, BDER00380_0101, BDER00381_0101, BDER00382_0101, BDER00383_0101, BDER00384_0101*), Atiama-Nurbē et al. leg. (dung-baited pitfall traps); 4 ô, 1 \%, Saint-Gilles, route du Théâtre (alt. 70 m), 9.III.2012, 29.III.2012, 29.VIII.2012 (BDER00376_0101*, BDER00377_0101, BDER00378_0101), M. Jacquot et al. leg. (pitfall traps).

Distribution. - Reunion Island (new record), Seychelles, India (Malabar Coast, coasts of Odisha and Tamil Nadu, West Bengal), Sri Lanka, Thailand, Malaysia, Indonesia, Taiwan, Philippines.

Family Mimetidae Simon, 1881

Genus Ero C. L. Koch, 1836

Ero aphana (Walckenaer, 1802) (fig. 3)

Aranea aphana Walckenaer, 1802: 206.

Ero aphana (Walckenaer): Simon, 1881: 33; Harms \& Harvey, 2009: 268, fig. 26.A-E, 28.I.

Material examined. - Reunion Island. 1 đે, Saint-Pierre, Mont-Vert-les-Bas (alt. 360 m), 27.VIII.2013 (BDER00373_01*), M. Jacquot et al. leg. (suction sampling on herbaceous layer).

Distribution. - Europe to Northwest China, Reunion Island (new record), Southeast Asia, Australasia, St. Helena.
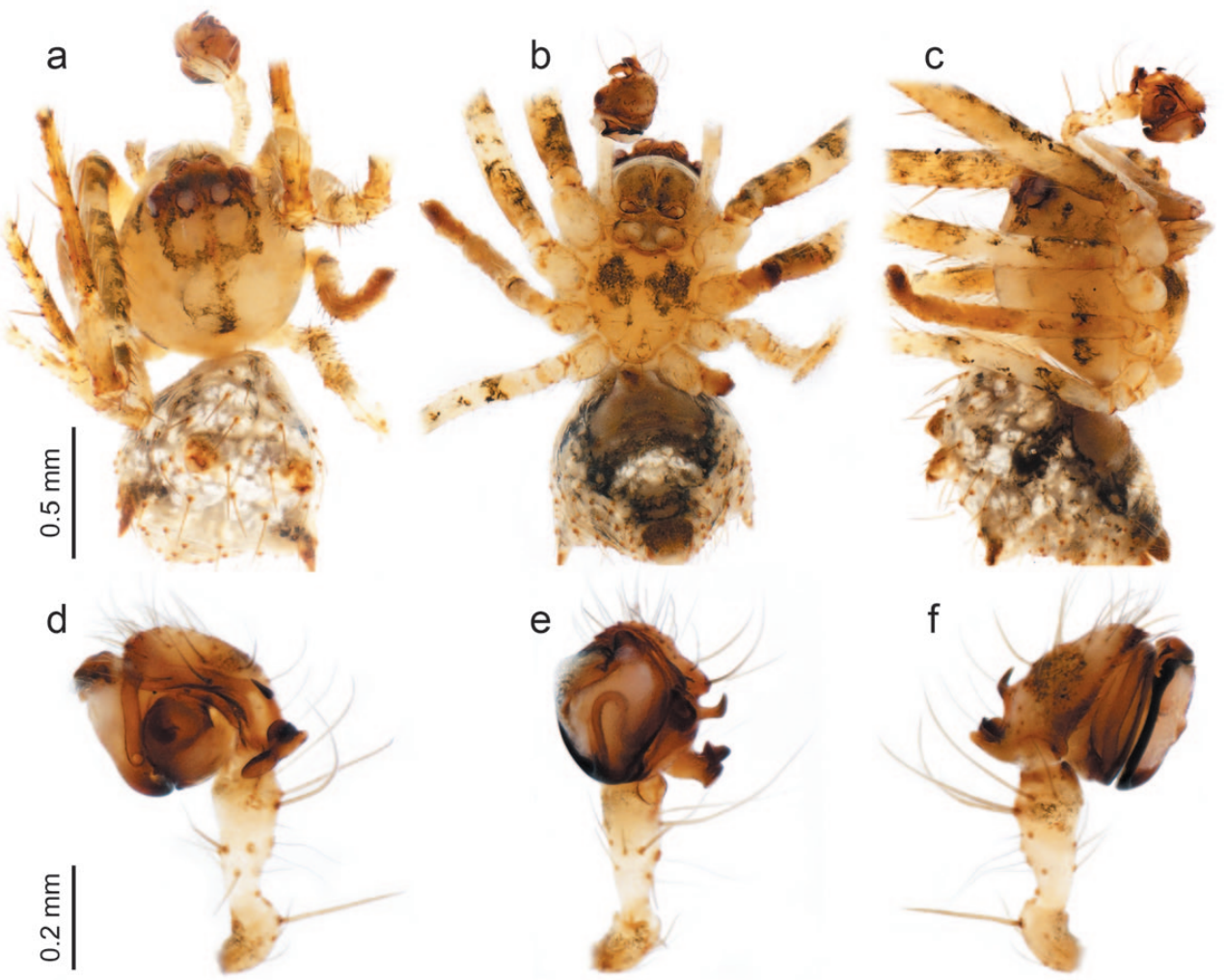

Fig. 3. - Ero aphana (Walckenaer), ô. - a, Dorsal view. - b, Ventral view. - c, Lateral view. - d, Palp, retrolateral view. - e, Palp, ventral view. - f, Palp, prolateral view. 
Family Miturgidae Simon, 1886

Genus Palicanus Thorell, 1897

Palicanus caudatus Thorell, 1897 (fig. 4)

Palicanus caudatus Thorell, 1897: 227; Deeleman-Reinhold, 2001: 220, fig. 266-271; SAaristo, 2002: 16, fig. 41-46; SAARISTO, 2010: 90, fig. 16.1-5.

Material examined. - Reunion Island. 4 స̃, 1 †, Saint-Gilles, route du Théâtre (alt. 70 m), 7.III.2012, 21.VIII.2013 (BDER00406_0101, BDER00407_0101*, BDER00408_0101, BDER00431_0101); $1 \hat{\jmath}$, 1 ㅇ, Saint-Gilles (alt. 90-110 m), 7.III.2012, 14.IIII.2012 (BDER00404_0101*, BDER00405_0101); all Jacquot et al. leg. (pitfall traps).

Distribution. - Reunion Island (new record), Seychelles, Lombok (island of south Indonesia), Hainan (island of south China) and Myanmar.

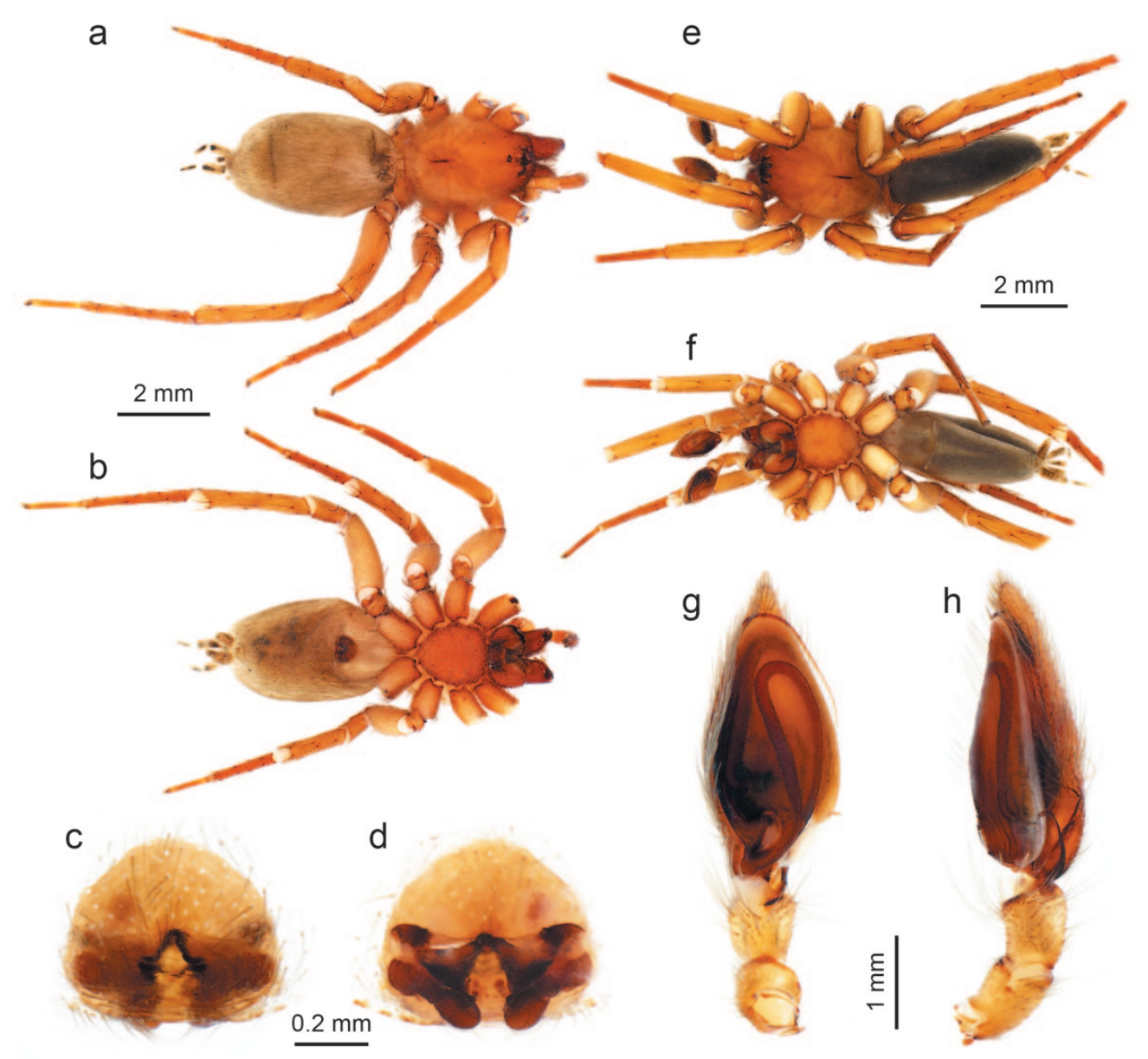

Fig. 4. - Palicanus caudatus Thorell. - a-d, o : a, dorsal view; b, ventral view; c, epigyne; d, vulva. - e-h, §’: e, dorsal view; f, ventral view; g, palp, ventral view; h, palp, retrolateral view. 
Family Oecobiidae Blackwall, 1862

Genus Oecobius Blackwall,1862

Oecobius marathaus Tikader, 1962 (fig. 5)

Oecobius marathaus Tikader, 1962: 684, fig. 2a-b.

Oecobius reefi Saaristo, 1978: 104, figs. 46-51.

Maitreja marathaus (Tikader): SAARISTO, 1997: 71, fig. 1-4; SAARISTO, 2010: 106, fig. 21.1-6.

Material examined. - Reunion Island. 4 đิ, Saint-Gilles, cap de la Houssaye (alt. 80 m), 19.VIII.2014 (BDER00374_01*), Jacquot et al. leg. (pitfall trap); 1 đ, 4 + , Saint-Pierre, chemin de l'Irat (alt. 160 m), 29.IV.2015, 9.IX.2015 (BDER00434_0101, BDER00375_0101*), B. Derepas leg. (night collecting).

Distribution. - Pantropical, recorded in Seychelles and newly recorded from Reunion Island.

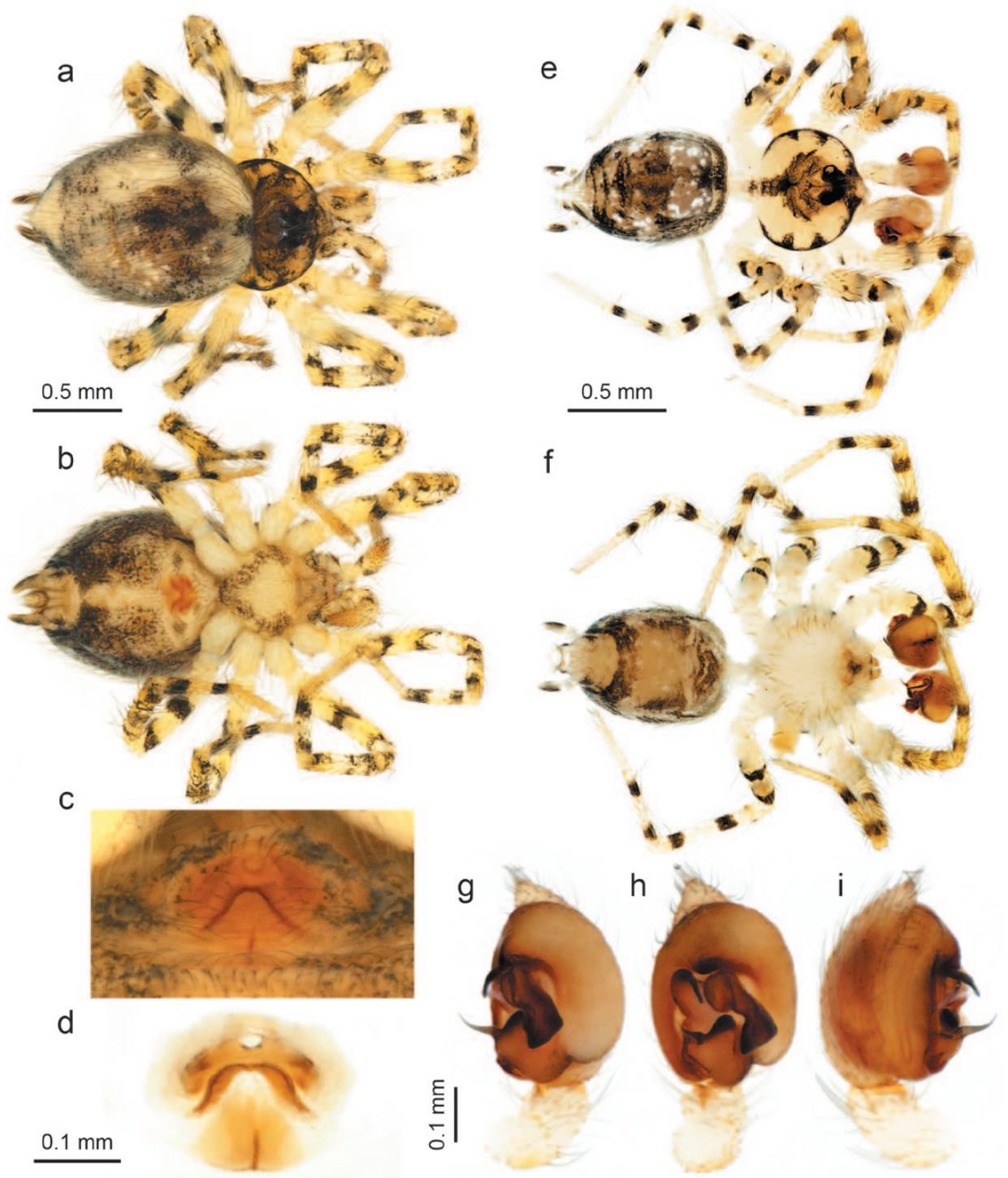

Fig. 5. - Oecobius marathaus Tikader. - a-d, o : a, dorsal view; b, ventral view; c, epigyne; d, vulva. - e-i, ð̊: e, dorsal view; f, ventral view; $g$, palp, retrolateral view; h, palp, ventral view; i, palp, prolateral view. 


\section{Family Phrurolithidae Banks, 1892}

Genus Orthobula Simon, 1897

Orthobula impressa Simon, 1897 (fig. 6)

Orthobula impressa Simon, 1897: 498; SAARISTO, 2002: 14, fig. 33-38; SAARISTO, 2010: 61, fig. 6.11-16.

Material examined. - Reunion Island. 1 , Plaine des Cafres, chapelle Notre-Dame-de-la-Paix (alt. 1635 m), 5.XII.2014 (BDER00390_0101*), B. Derepas leg.; 1 ô, 2 ㅇ, Étang-Salé (alt. 110 m), VIII.2014, (BDER00385_0101, BDER00386_0101, BDER00387_0101), M. Jacquot et al. leg. (suction sampling and pitfall trap); 4 o , 4 क , Saint-Gilles (alt. 70-110 m), VIII.2014 (BDER00391_01, BDER00392_0101, BDER00393_0101, BDER00394_0101, BDER00395_0101, BDER00396_0101, BDER00397_01), Jacquot et al. leg. (suction sampling and pitfall trap); $1 \overline{0}, 2$, , Saint-Paul, Piton-Défaud (alt. $11 \overline{0}$ ), VIII.2014 (BDER00398 0101*, BDER00399 0101, BDER00400 0101, BDER00401 01), Jacquot et al. leg. (suction sampling and pitfall traps); $1 \hat{\jmath}, 1$ +, Saint-Pierre, Pierrefond (alt. 60 m), VIII.2014 (BDER00402_0101, BDER00403_01), Jacquot et al.; 2 ㅇ, Saint-Pierre, Mont-Vert-les-Bas (alt. 360 m), VIII.2014 (BDER00388_0101, BDER00389_0101), Jacquot et al. leg. (pitfall traps).

Distribution. - Reunion Island (new record), Seychelles, Maharashtra (India), Sri Lanka and West Sumatra.

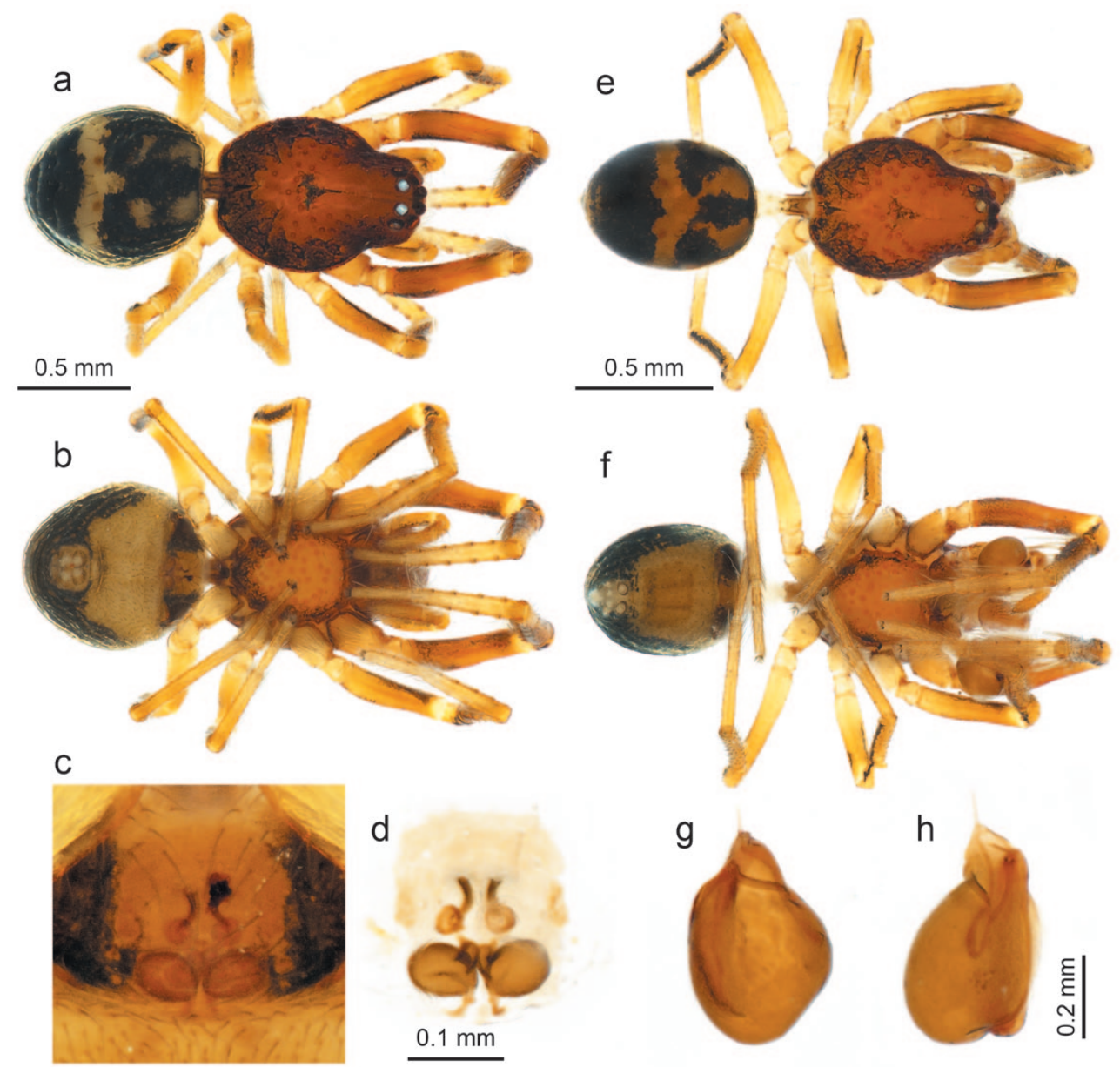

Fig. 6. - Orthobula impressa Simon. - a-d, ๆ: a, dorsal view; b, ventral view; c, epigyne; d, vulva. - e-h, đ̃: e, dorsal view; f, ventral view; g, bulb, ventral view; h, bulb, retrolateral view. 
Family Zodariidae Thorell, 1881

Genus Tropizodium Jocqué \& Churchill, 2005

Tropizodium peregrinum Jocqué \& Churchill, 2005 (fig. 7)

Tropizodium peregrinum Jocqué \& Churchill, 2005: 4, figs. 1-13.

Material examined. - Reunion Island. 25 วิ, 17 ค , Saint-Gilles (alt. 70-110 m), VIII.2014 (BDER00409 01, BDER00410 01, BDER00411 01, BDER00412 01, BDER00413 01, BDER00414 0101, BDER00415 01, BDER00416 0101, BDER00417 01, BDER00418 01, BDER00419 0101, BDER00420 01, BDER00421_0101, BDER00422_0101, BDER00423_01, BDER00424_01, BDER00425_01, BDER00426_01, BDER00427_01), Jacquot et al. leg. (pitfall traps); 4 ō, $\overline{5}$ o, idem, 21.VIII. 2013 (MRAC_243319, MRAC_243320,
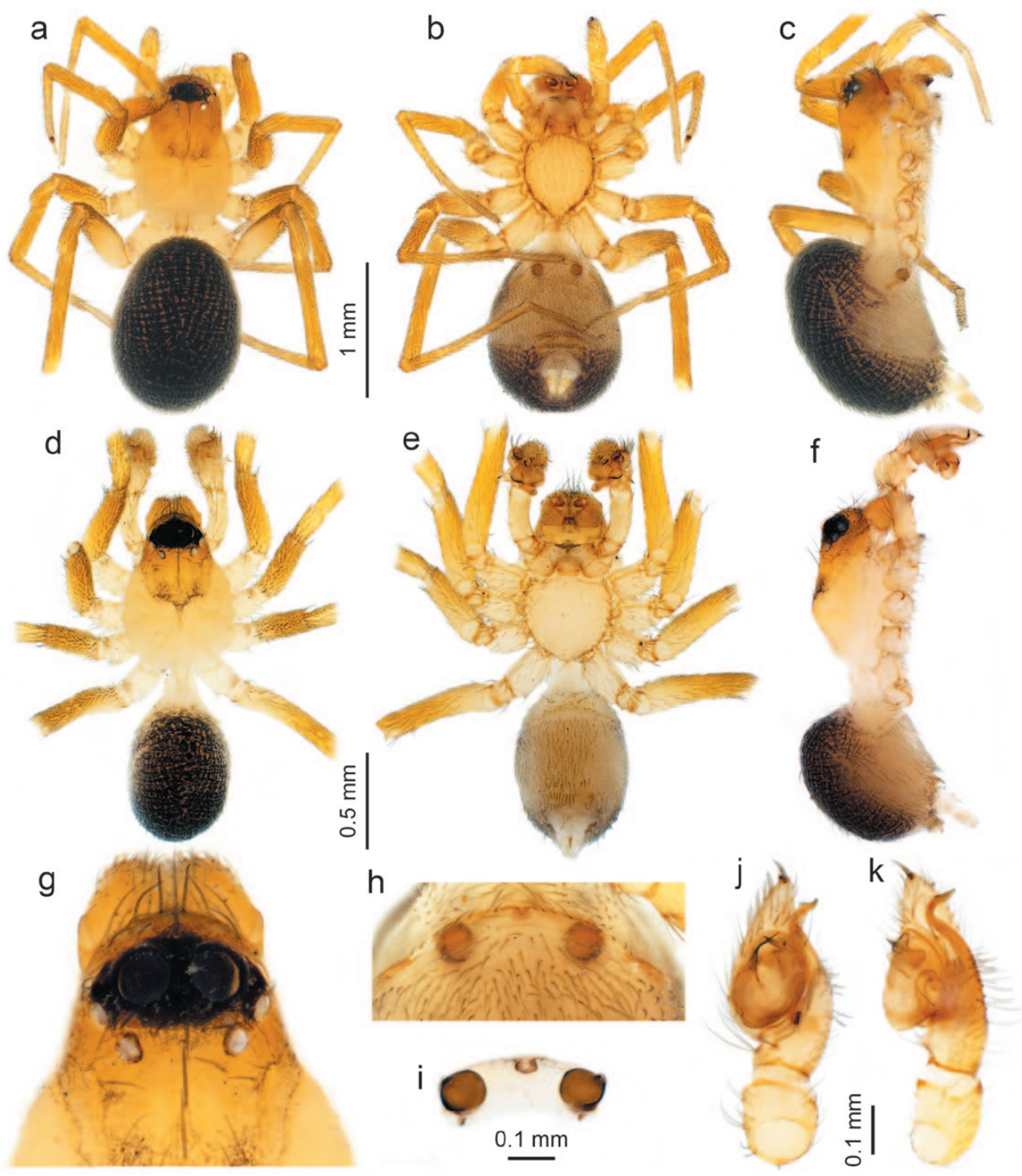

Fig. 7. - Tropizodium perigrinum Jocqué \& Churchill. - a-c, ,, : a, dorsal view; b, ventral view; c, lateral view. - d-f, $\jmath^{\lambda}$ :

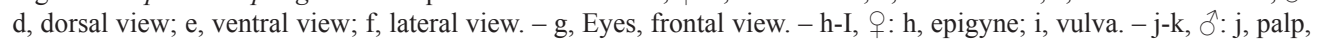
ventral view; $\mathrm{k}$, palp, retrolateral view. 
MRAC_243322 to MRAC_243323); 1 đ̂, Saint-Paul, Piton-Défaud (alt.110 m), 29.VIII.2012 (MRAC_243321); $4{ }^{\Uparrow}$, Saint-Pierre,Pierrefond(alt.60m), VIII.2014(BDER00428_01,BDER00429_01*,BDER00430_0101), Jacquot et al. leg. (pitfall traps); 1 ô, idem, 21.VIII.2013 (MRAC_243325); 2 ôे, 1 o, Saint-Pierre, chemin de l'Irat (alt. 160 m), 15.VIII.2014, IV.2015, (BDER00432_0101, BDER00433_0101), B. Derepas leg. (pitfall traps).

Distribution. - Reunion Island (new record) and Northern Territory (Australia).

\section{Discussion}

Spider fauna on Reunion Island counts now 31 families. Among the seven species newly recorded in Reunion, five have been previously recorded from Seychelles. Creugas gulosus and Oecobius marathaus have a pantropical distribution. For Orthobula impressa (known from Oriental region), Palicanus caudatus and Oedignatha scrobiculata (known from OrientalAustralian region), Reunion is the southwestern-most locality. Ero aphana has one of the wider distributions, but isn't known from Seychelles. Lastly, Tropizodium peregrinum was only known from type locality in Darwin (Australia) and in Reunion. This species now shows an unusual distribution among spiders.

ACKNOWLEDGEMENTS. - We dedicate this article to Jean-Claude Ledoux, for his outstanding work on spiders, for his kindness and his help on species identification. We are grateful to Arnaud Henrard and Rudy Jocqué for their contribution in the identification of Zodariidae species. We thank Sophie Gasnier and Grégory Cazanove for the organisation of the identification worshop with J.-C. Ledoux. We thank Mickaël Tenailleau, C. Ajaguin Soleyen, M.-L. Moutoussamy, S. Plessix, C. Baltzer and L. Müller for their assistance during sampling and A. Franck for his help in the photographic work. The authors greatly acknowledge the Plant Protection Platform (3P, IBISA). We extend our gratitude to the Ministry of Agriculture, Food, Fisheries, Rural Affairs and Spatial Planning, which funded the BIOPHYTO project via the Trust Account for Agricultural and Rural Development (CASDAR). This work was also funded by the European Agricultural Fund for Rural Development (EAFRD), by the Conseil Régional de la Réunion, by the Conseil Général de la Réunion and by the Centre de Coopération internationale en Recherche agronomique pour le Développement (CIRAD).

\section{REFERENCES}

Agnarsson I. \& Kuntner M., 2012. - The Generation of a Biodiversity Hotspot: Biogeography and Phylogeography of the Western Indian Ocean Islands (pp. 33-82). In : Anamthawat-Jonsson K. (ed), Current Topics in Phylogenetics and Phylogeography of Terrestrial and Aquatic Systems. Rijeka : In Tech, $114 \mathrm{p}$.

Deeleman-Reinhold C. L., 2001. - Forest spiders of South East Asia: with a revision of the sac and ground spiders (Araneae: Clubionidae, Corinnidae, Liocranidae, Gnaphosidae, Prodidomidae and Trochanterriidae). Leiden : Brill, $591 \mathrm{p}$.

Harms D. \& Harvey M. S., 2009. - Australian pirates: systematics and phylogeny of the Australasian pirate spiders (Araneae: Mimetidae), with a description of the Western Australian fauna. Invertebrate Systematics, $23: 231-280$.

Jocqué R. \& ChuRchill T. B., 2005. - On the new genus Tropizodium (Araneae: Zodariidae), representing the femoral organ clade in Australia and the Pacific. Zootaxa, 944:1-10.

Ledoux J.-C., 2004. - Araignées de l'île de La Réunion : I Hahniidae, Ctenidae, Thomisidae et Clubionidae (Araneae). Revue Arachnologique, $14:$ 159-191.

Ledoux J.-C. \& Hallé N., 1995. - Araignées de l'île Rapa (îles Australes, Polynésie). Revue Arachnologique, $11: 1-15$.

Lopez A., 1990. - Contribution à l'étude des araignées réunionnaises : note préliminaire. Bulletin de la Société Sciences Nat, 67 : 13-22.

Platnick N. I., Dupérré N., Ott R. \& Kranz-Baltensperger Y., 2011. - The Goblin Spider Genus Brignolia (Araneae, Oonopidae). Bulletin of the American Museum of Natural History, 349 : 1-131.

Quilici S., Vincenot D., Franck A., Ohm P., Kreiter S., Delvare G., Kassebeer C. \& Ledoux J.-C., 2003. - Les auxiliaires des cultures fruitières à l'île de La Réunion. Saint-Denis : CIRAD, 168 p. 
RAVEN R. J., 2015. - A revision of ant-mimicking spiders of the family Corinnidae (Araneae) in the Western Pacific. Zootaxa, 3958 (1) : 1-258.

SaARisto M. I., 1978. - Spiders (Arachnida, Araneae) from the Seychelles islands, with notes on taxonomy. Annales Zoologici Fennici, 15 : 99-126.

1997. - Oecobiids of the granitic islands of Seychelles (Araneae, Oecobiidae). Phelsuma, $5: 69-71$. 2002. - New species and interesting new records of spiders from Seychelles (Arachnida, Araneaea). Phelsuma, $10: 1-31$.

2010. - Araneae (p. 8-306). In : Gerlach J. \& Marusik Y (eds), Arachnida and Myriapoda of the Seychelles islands. Manchester : Siri Scientific Press, 435 p.

Schmidt G. E. W. \& JocquÉ R., 1983. - Spinnen von der Insel Réunion (Araneae). Revue Zoologique Africaine, 97 : 353-364.

Simon E., 1881. - Les arachnides de France. Tome 5 (1). Paris : Librairie Encyclopédique de Roret, 180 p. +25 pl.

— 1897. - Études arachnologiques. $27^{e}$ mémoire. XLII. Descriptions d'espèces nouvelles de l'ordre des Araneae. Annales de la Société entomologique de France, 65 : 465-510.

Thorell T., 1878. - Studi sui ragni Malesi e Papuani. II. Ragni di Amboina raccolti Prof. O. Beccari. Annali del Museo Civico di Storia Naturale di Genova, 13 : 1-317.

— 1881. - Studi sui Ragni Malesi e Papuani. III. Ragni dell'Austro Malesia e del Capo York, conservati nel Museo civico di storia naturale di Genova. Annali del Museo Civico di Storia Naturale di Genova, $17: 1-727$.

1897. - Viaggio di Leonardo Fea in Birmania e regioni vicine. LXXIII. Secondo saggio sui Ragni birmani. I. Parallelodontes. Tubitelariae. Annali del Museo Civico di Storia Naturale di Genova, (2) $17: 161-267$.

TIKADER B. K. 1962. - Studies on some Indian spiders (Araneae: Arachnida). Journal of the Linnean Society of London, Zoology, 44 : 561-584.

Vinson A., 1863. - Aranéides des îles de la Réunion, Maurice et Madagascar. Paris : Librairie encyclopédique de Roret, $337 \mathrm{p}$.

WALCKenAer C. A. 1802. - Faune parisienne, insectes, ou, Histoire abrégée des insectes des environs de Paris : classés d'après le système de Fabricius ; précédée d'un discours sur les insectes en général, pour servir d'introduction à l'étude de l'entomologie. Tome II. Paris : Dentu, XXII + 438 p. 